\title{
COMPOSTOS FENÓLICOS PRODUZIDOS A PARTIR DE MICROALGAS
}

\author{
D. P. WEBER ${ }^{1}$, T.C. NASCIMENTO ${ }^{1}$, M. T. BARCIA ${ }^{1}$, E. JACOB-LOPES ${ }^{1}$, L.Q. ZEPKA ${ }^{1}$ \\ ${ }^{1}$ Universidade Federal de Santa Maria, Departamento de Ciência e Tecnologia em alimentos. \\ E-mail para contato: lqz@pq.cnpq.br
}

\begin{abstract}
RESUMO - As microalgas tem despertado cada vez mais atenção pela sua capacidade em sintetizar inúmeros metabólitos bioativos de interesse. Nesse sentido, o objetivo do trabalho foi avaliar o conteúdo fenólico bem como o potencial antioxidante de Scenedesmus obliquus CPCC05 e Phormidium autmnalle. As biomassas foram produzidas em fotobiorreator de coluna de bolhas acoplado a uma plataforma de iluminação, operando de forma intermitente com alimentação de 1,5 L de meio BG11, concentração inicial de inoculo de $100 \mathrm{mg} . \mathrm{L}^{-1}$, temperatura de 25 ${ }^{\circ} \mathrm{C}$, aeração de 1 volume de ar por volume de meio por minuto (VVM) através da injeção de ar enriquecido com $15,0 \%$ de dióxido de carbono (v/v), a relação volume de meio circundante na plataforma de iluminação e área escura foi de $20 \%$ e intensidade luminosa de 13000 Lux. O conteúdo fenólico foi extraído das biomassas por meio de agitação orbital com metanol e determinado através de reagente de Folin-Ciocalteu. A atividade antioxidante foi estabelecida através da capacidade do extrato fenólico em desativar espécies reativas de oxigênio. Com base nos resultados obtidos, a microalga Scenedesmus obliquus CPCC05 apresentou maior conteúdo fenólico total $\left(871,2 \mu \mathrm{g}_{\text {EAG.g }}{ }^{-1}\right)$ bem como maior potencial antioxidante $\left(23,5 \mu \mathrm{Mol} \mathrm{TE} . \mathrm{g}^{-1}\right)$ em relação a Phormidium autumnale $\left(552,6 \mu \mathrm{g} \mathrm{EAG.g}^{-1}\right.$ e 16,3 $\mu \mathrm{Mol}$ TE. $\left.\mathrm{g}^{-1}\right)$. Deste modo, sugere-se que essa microalga pode ser explorada como uma via natural de produção compostos fenólicos.
\end{abstract}

\section{INTRODUÇÃO}

As microalgas são micro-organismos promissores, uma vez que são constituídas por uma variedade de biomoléculas de interesse comercial, tais como aminoácidos, ácidos graxos, carotenoides, clorofila e compostos fenólicos (Zepka et al., 2008; Jacob-Lopes et al., 2007; Klejdus et al., 2010; Queiroz et al., 2011; Rodrigues et al. 2014; Maadane et al., 2015; Maroneze et al, 2016).

Dentre esses microrganismos as clorofíceas e cianobactérias estão entre as linhagens que tem sido fortemente exploradas em função do seu potencial produção de biomassa associado a esses valiosos compostos celulares, os quais podem ser amplamente aplicados como insumos intermediários e produtos finais de processos relacionados à bioenergia, alimentação e farmacêuticos (Zepka et al., 2008; Jacob-Lopes et al., 2010; Zepka et al., 2010; Queiroz et al., 2011; 2014; Rodrigues et al. 2015; Santos et al., 2016; Maroneze et al, 2016).

Muitas pesquisas tem sido amplamente direcionadas para exploração de biocompostos microalgais (Zepka et al., 2008; Jacob-Lopes et al., 2007; Queiroz et al., 2011; Rodrigues et al. 
2014; Rodrigues et al. 2015; Maroneze et al, 2016), no entanto compostos fenólicos ainda são pouco explorados, embora sua presença em biomassa microalgal já tenha sido considerada há algum tempo (Klejdus et al., 2009).

Compostos fenólicos são uma classe importante de antioxidantes, uma vez que são capazes de impedir a oxidação lipídica por eliminação direta de espécies reativas de oxigênio entre outras funções, por conta disso assumem importante papel em termos de saúde humana ou aplicações tecnológicas (Pietta, 2000).

Com base no exposto, o presente estudo teve por objetivo avaliar o conteúdo compostos fenólicos totais presente nas biomassas de Scenedesmus obliquus CPCC05 e Phormidium autmnalle, bem como determinar seu potencial antioxidante.

\section{MATERIAL E MÉTODOS}

\subsection{Microrganismos, meio de cultura e produção de biomassa}

Culturas axênicas de Scecnedesmus obliquus CPCC05 e Phormidium autumnale foram propagadas e mantidas em meio de BGN sintético contendo ágar-ágar solidificado (20 g.L $\left.\mathrm{L}^{-1}\right)$ (Rippka et al., 1979). As condições de incubação utilizadas foram de $25^{\circ} \mathrm{C}$ e intensidade luminosa constante de 1000 Lux.

A produção de biomassa foi realizada em fotobiorreator (Jacob-Lopes et al., 2014) integrando duas unidades principais de reação: um fotobiorreator de coluna de bolhas acoplado a uma plataforma de iluminação. $\mathrm{O}$ fotobiorreator operou em regime intermitente, alimentado com 1,5 L de meio BGN (Ripka et al., 1979). As condições experimentais foram: concentração inicial de inoculo de $100 \mathrm{mg} . \mathrm{L}^{-1}$, temperatura de $25^{\circ} \mathrm{C} \pm 1$, aeração de 1 volume de ar por volume de meio por minuto (VVM), através da injeção de ar enriquecido com 15,0\% de dióxido de carbono $(\mathrm{v} / \mathrm{v})$. A relação entre o volume de meio circundante na plataforma de iluminação e área escura foi de $20 \%$, além disso a intensidade luminosa de 13000 Lux permaneceu constante.

\subsection{Extração e quantificação dos compostos fenólicos totais}

A extração de compostos fenólicos foi realizada conforme metodologia proposta por Souza et al., (2011), com adaptações. 0,2 $\mathrm{g}$ de biomassa liofilizada foram homogeneizadas com $2 \mathrm{~mL}$ de metanol em agitador orbital a $25{ }^{\circ} \mathrm{C}$ durante $60 \mathrm{~min}$ a $200 \mathrm{rpm}$. A agitação foi interrompida aos 45 minutos, após foram adicionados $2 \mathrm{~mL}$ de metanol e a agitação foi retomada por mais $60 \mathrm{~min}$. $\mathrm{O}$ extrato foi filtrado e lavado três vezes com $10 \mathrm{~mL}$ de hexano para fins de purificação. $\mathrm{O}$ extrato purificado foi clarificado para eliminação de proteínas com hidróxido de bário $0,1 \mathrm{M}$ e sulfato de zinco 5\%, após submetido a centrifugação durante $10 \mathrm{~min}$ a $3000 \mathrm{rpm}$. O sobrenadante foi removido, filtrado através de membrana de 0,22 $\mu \mathrm{m}$ (Millipore) e a concentração ajustada em balão volumétrico. Em seguida, os extratos foram encaminhados para determinação do conteúdo total de fenólicos bem como sua capacidade antioxidante.

O teor de fenólicos totais foi estimado pelo método de Folin-Ciocalteu adaptado de Singleton et al. (1999). $2,5 \mathrm{~mL}$ de amostra foram adicionados a $0,5 \mathrm{~mL}$ de reagente de FolinCiocalteu dilúido (1:10). Após $5 \mathrm{~min}$, adicionou-se $2 \mathrm{~mL}$ de carbonato de sódio a 7,5\%. Decorridas $2 \mathrm{~h}$ de incubação à temperatura ambiente e ao abrigo da luz, mediu-se a absorbância a $760 \mathrm{~nm}$. A quantificação foi feita por calorimetria, por meio de curva de calibração de sete 
pontos, construída com ácido gálico $(170,1 \mathrm{mM})$. Os resultados foram expressos em equivalente de ácido gálico por grama de peso seco de microalga (mg GAE. $\left.{ }^{-1}\right)$ e referem-se ao valor médio de três repetições.

\subsection{Potencial antioxidante dos extratos fenólicos totais}

A capacidade antioxidante dos extratos fenólicos determinada conforme o método proposto por $\mathrm{Ou}$ et al., 2013 para amostras hidrofílicas, com adaptações. $25 \mu \mathrm{L}$ de extrato fenólico ou tampão fosfato (branco) foram colocados em microplaca de 96 poços contendo 150 $\mu \mathrm{L}$ de fluoresceína $(81 \mathrm{mM})$ em tampão fosfato $(75 \mathrm{mM} \mathrm{e} \mathrm{pH} \mathrm{7,4).} \mathrm{A} \mathrm{mistura} \mathrm{foi} \mathrm{pré-incubada}$ durante 10 min a $37^{\circ} \mathrm{C}$ seguida da adição de $25 \mu \mathrm{L}$ de AAPH $(19 \mathrm{mM})$. O sinal de fluorescência foi registado cada minuto até 80 min num SpectraMax M5 (Molecular Devices Corp, Los Angeles-CA USA) ou até atingir $0,5 \%$ do sinal de fluorescência inicial. A fluorescência foi monitorizada no comprimento de onda de emissão de $520 \pm 20 \mathrm{~nm}$ com excitação de $485 \pm 20$ nm. Registou-se a fluorescência relativa em função do tempo e calculou-se a área sob curva (AUC) da amostra e do branco. Os resultados foram expressos em $\mu$ mol trolox equivalente por grama de peso seco de biomassa microalgal $\left(\mu \mathrm{mol} \mathrm{TE} . \mathrm{g}^{-1}\right)$ e referem-se ao valor médio de três repetições.

\subsection{Análise estatística}

A análise de variância (ANOVA) e o teste de Tukey $(\mathrm{p}<0,05)$ foram utilizados para verificar as diferenças entre as microalgas. As análises foram realizadas com o software Statistica 7.0 (StatSoft, Tulsa-OK, EUA).

\section{RESULTADOS E DISCUSSÕES}

O conteúdo de fenólicos das biomassas microalgais está apresentado na Tabela 1. Os teores diferiram significativamente $(\mathrm{p}<0,05)$, a microalga Scenedesmus obliquus CPCC05 $\left(871,20 \mu \mathrm{g}\right.$ EAG. $\left.\mathrm{g}^{-1}\right)$ exibiu maior teor de fenólicos quando comparada a Phormidium autumnale (552,6 $\mu \mathrm{g}$ EAG. $\left.\mathrm{g}^{-1}\right)$. No entanto, o conteúdo encontrado para as duas microalgas foi inferior ao relatado para a maioria das microalgas analisadas em estudo realizado por Goiris et al. 2012 (1090 a $\left.4570 \mu \mathrm{g} \mathrm{EAG.g}{ }^{-1}\right)$.

A ausência de proteínas nos extratos fenólicos do presente estudo pode justificar o limiar mínimo destes compostos em comparação com a literatura, uma vez que a estrutura química das proteínas através de reações de redução sob o reagente de Folin-Ciocalteu produz um composto que apresenta absorção máxima na faixa de $760 \mathrm{~nm}$, a qual é mesma faixa de absorção para fenólicos (Agbor et al., 2014).

Tabela 2. Potencial antioxidante dos extratos fenólicos microalgais.

\begin{tabular}{lc} 
Microalgae & $\mu{\mathrm{Mol} \mathrm{TE} . \mathrm{g}^{-1}}^{\mathrm{n}}$ \\
\cline { 1 - 1 } Scenedesmus obliquus CPCC05 & $23,5^{\mathrm{a}} \pm 0,1$ \\
Phormidium autumnale & $16,3^{\mathrm{b}} \pm 0,0$ \\
\hline As médias com diferentes sobrescritos são significativamente diferentes $(\mathrm{p}<0,05)$ pelo teste de Tukey.
\end{tabular}


A capacidade de eliminação das espécies reativas de oxigênio (EROs) no presente estudo foi medida por monitoramento do efeito de antioxidantes sobre o decaimento da fluorescência, o qual é causado pela oxidação da sonda de fluoresceína induzida por EROs geradas pela decomposição térmica de AAPH à $37^{\circ} \mathrm{C}$ (OU et al. 2013). Diante disso, pode-se observar na Figura 1 que o extrato fenólico da $S$. obliquus foi capaz de manter a fluorescência da sonda mais estável quando comparado ao extrato da $P$. autumnale, evidenciando assim maior potencial antioxidante $\left(23,48 \pm 0.13 \mu \mathrm{Mol} \mathrm{TE} . \mathrm{g}^{-1}\right)$ conforme é demonstrado na Tabela 2, além disso o teste de Tukey $(\mathrm{p}<0,05)$ mostrou uma diferença é significativa entre os teores de fenólicos encontrados para as duas algas estudadas. Este resultado corrobora com o conteúdo fenólico encontrado nas microalgas (Tabela 1).

De acordo com estudos realizados por Cai et al. (2004), amostras que apresentarem os

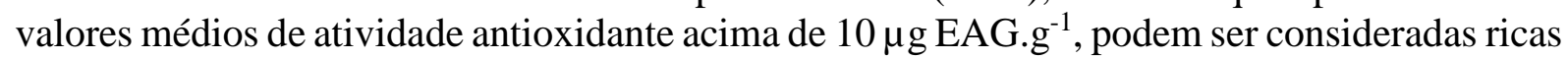
em antioxidantes. Com isso, os valores obtidos no presente estudo evidenciam o potencial antioxidante destes compostos na biomassa das microalgas exploradas.

Figura 1. Decaimento da fluorescência de fluoresceína induzida por radicais peroxil na presença de extrato fenólico de Scenedesmus obliquus CPCC05 e Phormidium autumnale.

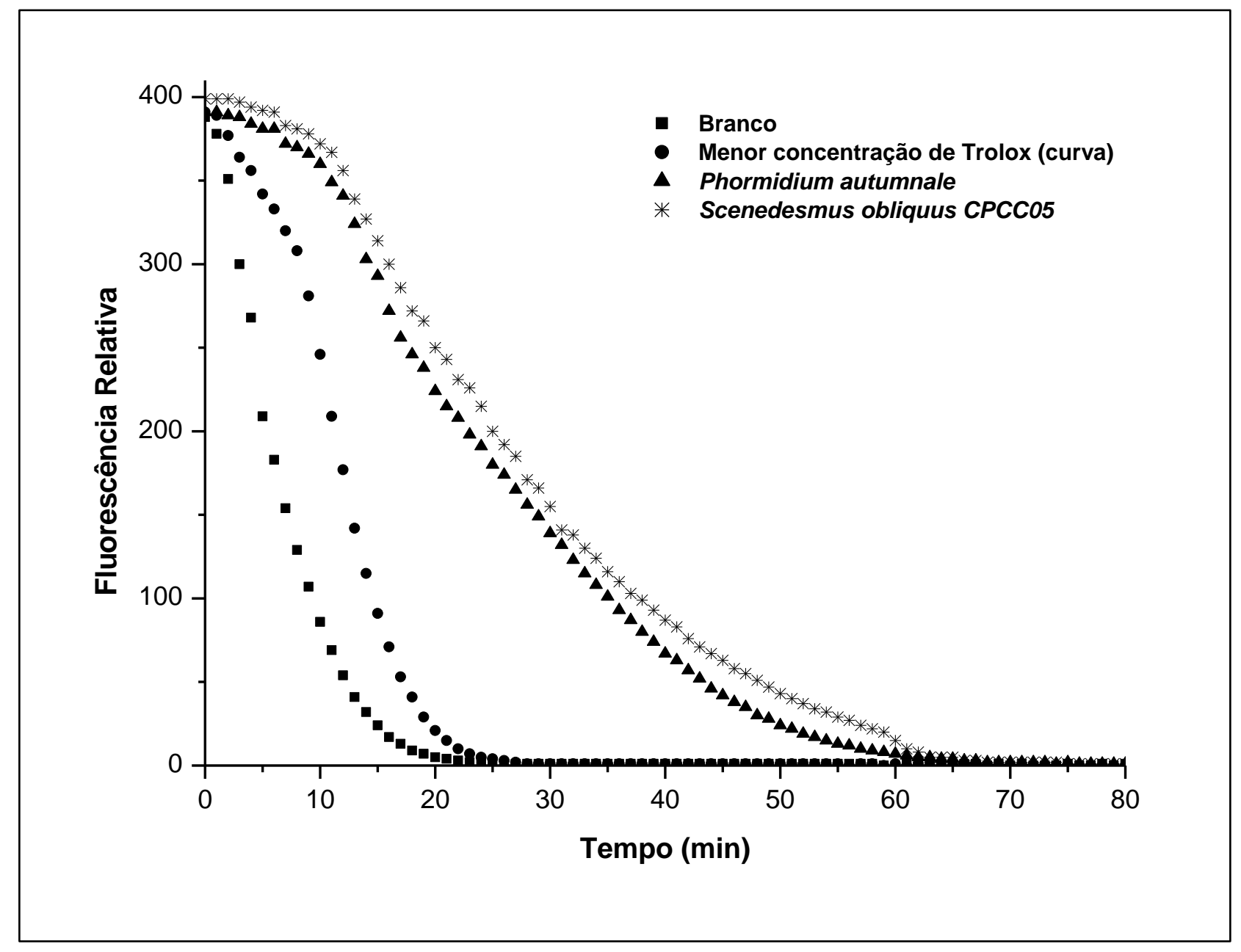

Tabela 2. Potencial antioxidante dos extratos fenólicos microalgais. 


\begin{tabular}{lc} 
Microalgae & $\mu{\mathrm{Mol} \mathrm{TE} . \mathrm{g}^{-1}}^{\mathrm{n}}$ \\
\hline Scenedesmus obliquus CPCC05 & $23,5^{\mathrm{a}} \pm 0,1$ \\
Phormidium autumnale & $16,3^{\mathrm{b}} \pm 0,0$ \\
\hline
\end{tabular}

As médias com diferentes sobrescritos são significativamente diferentes $(\mathrm{p}<0,05)$ pelo teste de Tukey.

\section{CONCLUSÃO}

Com base nos resultados obtidos, a microalga Scenedesmus obliquus CPCC05 apresentou maior conteúdo de fenólicos totais bem como maior potencial antioxidante quando comparada a Phormidium autumnale.

\section{REFERÊNCIAS}

AGBOR, G. A.; VINSON, J. A.; DONNELLY P. E. Folin-Ciocalteau Reagent for Polyphenolic Assay. Int. J Food Sci, Nutr and Diet, v.3, n.8, p.147-156.

CAI, Y.; LUO, Q.; SUN, M.; CORKE, H. Antioxidant activity and phenolic compounds of 112 traditional Chinese medicinal plants associated with anticancer. Life Sci, v.74, p.21572184, 2004.

GOIRIS, K.; MUYLAERT. K.; FRAEYE, I.; FOUBERT, I.; DE BRABANTER, J.; DE COOMAN, L. Antioxidant potential of microalgae in relation to their phenolic and carotenoid content. J. Appl. Phycol., v. 24, p.1477-1486, 2012.

JACOB-LOPES, E.; ZEPKA, L.Q.; PINTO, L.A.A.; QUEIROZ, M.I. Characteristics of thinlayer drying of the cyanobacterium Aphanothece microscopica Nägeli. Chem. Eng. Process, v. 46, p. 63-69, 2007.

JACOB-LOPES, E.; SCOPARO, C. H. G.; QUEIROZ, M. I.; FRANCO, T. T. Biotransformations of carbon dioxide in photobioreactors, Energy Convers. Manage, v. 51, p. 894-900, 2010.

JACOB-LOPES, E. et al. Bioprocess for the conversion of carbon dioxide from industrial emissions, bioproducts, uses thereof and hybrid photobioreactor. BR Pat. 041028. 15 sep. 33p. 2014.

KLEJDUS, B.; KOPECKÝB, J.; BENEŠOVÁ, L.; VACEKA, J. Solid-phase/supercriticalfluid extraction for liquid chromatography of phenolic compounds in freshwater microalgae and selected cyanobacterial species. J. Chromatogr. A, v.1216, n.5, p.763$771,2009$.

MAADANE, A.; MERGHOUB, N.; AINANE, T.; EL ARROUSSI, H.; BENHIMA, R.; AMZAZI, S.; BAKRI, Y.; WAHBY, I. Antioxidant activity of some Moroccan marine microalgae: Pufa profiles, carotenoids and phenolic content. J. Biotechnol., v.215, n.10 p.13-19, 2015.

MARONEZE, M. M.; SIQUEIRA, S. F.; VENDRUSCOLO, R. G.; WAGNER, R.; MENEZES, C. R.; ZEPKA, L. Q.; JACOB-LOPES, E. The role of photoperiods on photobioreactors - A potential strategy to reduce costs. Bioresour. Technol., v.219, p.493-499, 2016. 
OU, B.; CHANG, T.; HUANG, D.; PRIOR, RL. Determination of Total Antioxidant Capacity by Oxygen Radical Absorbance Capacity (ORAC) Using Fluorescein as the Fluorescence Probe: First Action 2012.23. J. AOAC Int., v. 96, n. 6, p.1372-1376, 2013

PIETTA P. G. Flavonoids as antioxidants. J. Nat. Prod., v.63, p.1035-1042, 2000.

QUEIROZ, M. I.; HORNES, M. O.; SILVA-MANETTI, A. G.; JACOB-LOPES, E. Singlecell oil production by cyanobacterium Aphanothece microscopica Nägeli cultivated heterotrophically in fish processing wastewater. Applied Energy, v.88, n.10, p.34383443, 2011.

RIPPKA, R.; DERUELLES, J.; WATERBURY, J. B.; HERDMAN, M.; STAINER, R. Y. Generic assignments strain histories and properties of pure cultures of cyanobacteria. $J$. Gen. Microbiol., v. 111, n. 1, p.1-61, 1979.

RODRIGUES, D. B.; MENEZES, C.R.; MERCADANTE, A. Z.; LOPES, E.J.; ZEPKA, L. Q. Bioactive pigments from microalgae Phormidium autumnale. Food Res Int, v.77, n.2, p.273-279, 2015.

RODRIGUES, D. B.; FLORES, E.M.M.; BARIN, J.S.; MERCADANTE A.Z.; JACOBLOPES, E.; ZEPKA, Q.L. Production of carotenoids from microalgae cultivated using agroindustrial wastes. Food Res Int, v.65, p.144-148, 2014.

SANTOS, A. B.; FERNANDES, A. S.; WAGNER, R.; JACOB-LOPES, E.; ZEPKA, L. Q. Biogeneration of volatile organic compounds produced by Phormidium autumnale in heterotrophic bioreactor. J. Appl. Phycol, v.28, n.2, p.1561-1570, 2016.

SINGLETON, V. L.; ORTHOFER, R.; LAMUELA-RAVENTOS, R.M. Analysis of total phenols and other oxidation substrates and antioxidants by means of Folin-Ciocalteu reagent. Methods Enzymol, v.299, p.152-178, 1999.

SOUZA, M. M.; PRIETTO L.; RIBEIRO, A. C.; SOUZA, T. D.; BADIALE-FURLONG, E. Assessment of the antifungal activity of Spirulina platensis phenolic extract against Aspergillus flavus. Ciência e Agrotecnologia, Lavras, v.35, n.6, p.1050-1058,2011.

ZEPKA, L. Q.; JACOB-LOPES, E.; GOLDBECK, R.; QUEIROZ, M.I. Production and biochemical profile of the microalgae Aphanothece microscopica Nägeli submitted to different drying conditions. Chem. Eng. Process, v. 47, n. 8, p. 1311-1316, 2008.

ZEPKA, L. Q.; JACOB-LOPES, E.; GOLDBECK, R.; SOUZA- SOARES, L.A.; QUEIROZ, M.I. Nutritional evaluation of single-cell protein produced by Aphanothece microscopica Nägeli. Bioresour. Technol., v.101, n.18, p.7107-7111, 2010. 